\title{
EANM Focus 3: The International Conference on Molecular Imaging and Theranostics in Neuroendocrine Tumours-the consensus in a nutshell
}

\author{
Jolanta Kunikowska ${ }^{1}$ (D) Valentina Ambrosini ${ }^{2,3} \cdot$ Ken Herrmann ${ }^{4}$ \\ Published online: 24 February 2021 \\ (C) The Author(s), under exclusive licence to Springer-Verlag GmbH, DE part of Springer Nature 2021
}

The third edition of the EANM Focus Meeting series was held in Athens, Greece, at the end of January 2020. There we had the pleasure and honour of conducting the consensus meeting. As in previous years, the Focus Meeting, EANM's winter event, covered a single topic and provided a discussion platform for physicians with different specialization working in the field of neuroendocrine tumours (NET).

As the diagnosis and treatment of NET patients is truly multidisciplinary and with nuclear medicine playing a significant role in it, Focus 3 was planned in close collaboration with the European Neuroendocrine Tumour Society (ENETS). This involved recruiting a large faculty of experts covering all the specialties involved in management to lecture and participate in this consensus meeting. Although considered a rare disorder, the past decade has witnessed an increase in the prevalence of NET. Its multifaceted presentation and clinical behaviour render its management an object of active debate.

The aim of this gathering was to provide an in-depth overview and critical assessment of the latest developments in the management of neuroendocrine tumours and the direction it will take in the future. Its special focus was on the role of imaging in general and nuclear medicine in particular. The

This article is part of the Topical Collection on Editorial

Jolanta Kunikowska

jolanta.kunikowska@wum.edu.pl

1 Nuclear Medicine Department, Medical University of Warsaw, ul. Banacha 1 a, 02-097 Warsaw, Poland

2 IRCSS, Azienda Ospedaliero-Universitaria di Bologna, Bologna, Italy

3 Nuclear Medicine, Department of Experimental, Diagnostic and Specialty Medicine, Alma Mater Studiorum University of Bologna, Bologna, Italy

4 Department of Nuclear Medicine, Universitätsklinikum, Essen, Germany goal was to obtain a multidisciplinary consensus on the current state of practice and to make expert recommendations on how to advance the field towards having a clinical impact.

The Delphi technique was used in an attempt to achieve consensus. The Delphi methodology is a structured communication technique which was developed as a systematic and interactive method for the purpose of generating consensus. One of the most important points is the involvement of panellists based on their expertise and publication record. To build a well-balanced composition of panellists, the specialization, gender and country distribution was considered. The majority of experts came from Europe, but we also included experts from the USA and Australia. A total of 24 panellists came. Their specialisms included nuclear medicine (nine panellists), endocrinology (five panellists), medical oncology (three panellists), medical physics (one panellist), surgery (one panellist), radio pharmacy (one panellist), gastroenterology (one panellist), pathology (one panellist) and radiology (two panellists). A patient representative was also presented.

The experts answered questionnaires in three rounds. Two of the rounds took place before the meeting while the third and final round was held at the same time. The final round only considered those questions which had not achieved consensus in the first two rounds after the presentation of data and a moderated discussion.

Consensus was reached in 15 of 17 questions concerning imaging of NEN, 3 of 5 addressing imaging and therapy of PPGL, 8 of 13 questions regarding PRRT of NET, 2 of 3 addressing treatment and monitoring and 3 of 5 about future perspectives for nuclear medicine in NEN. In summary, we obtained agreement in 31 of 43 questions (72\%).

Despite the gathering of 24 experienced specialists in the diagnosis and treatment of NET and 3 days of debates and discussions, it is good that some issues remain unresolved. This reflects the many advances in medicine generally, in nuclear medicine in particular and in NET management across the past decade. It also highlights the need for further 
investigation and research into imaging and diagnostics especially and at the molecular level as well. However, it is important to mention that the overarching goal is to improve the treatment of patients. We hope that the results of the Focus 3 meeting will constitute a solid foundation for further improvements in the care of NET patients. The manuscript was recently published in the European Journal of Cancer [1].

Highlights of Focus 3:

- For NET diagnosis: $\left[{ }^{68} \mathrm{Ga}\right] \mathrm{Ga}-\mathrm{DOTA}-\mathrm{SSA}$ PET/CT and diagnostic $\mathrm{CT}$ are the mainstay

- $\left.\quad{ }^{18} \mathrm{~F}\right] \mathrm{FDG}$ in G3 NET, NEC, rapidly progressive cases or SSA-negative lesions

- For suspected extra-adrenal localization of PPGL: $\left[{ }^{68} \mathrm{Ga}\right] \mathrm{Ga}-\mathrm{DOTA}-\mathrm{SSA}$

- PRRT is indicated at first progression of G1-G2 GEP NET and selected NET G3

- PRRT as second line for GI-NET, if there is sufficient uptake in all lesions

The concept of face-to-face meetings between clinicians and imaging experts will be continued in order to provide an active discussion platform for the improvement of clinical management. The next Focus 4 event was initially planned for February 2021, but it has been postponed to February 2022 in Seville, Spain, due to the COVID pandemic. The chosen topic is "Imaging and Therapy in Haematological Tumours". Imaging of aggressive lymphoma was one of the key drivers of the $\left[{ }^{18} \mathrm{~F}\right] \mathrm{FDG}$ PET/CT success story in the past. Lugano and Deauville classifications set preliminary standards for standardized reporting and reproducible response assessment.

Attempts to extend nuclear medicine diagnostics beyond aggressive lymphoma have failed thus far. Furthermore, the promotion of radioimmunotherapy has not resulted in the success that was anticipated despite clinically convincing data. However, this chapter seems to have been rewritten recently owing to subsequent developments.

Planning of the Focus 5 meeting is already underway and the topic will be chosen soon.

\section{Declarations}

Informed consent Not applicable.

Ethics approval Institutional Review Board approval was not required because the paper is an editorial.

Conflict of interest The author Jolanta Kunikowska declares no conflicts of interest with the submitted work.

Valentina Ambrosini reports personal fees from ESMIT and AAA outside the submitted work and is a member of the ENETS advisory board, ESMO faculty staff for NET and the scientific board of ITANET.

Ken Herrmann reports personal fees from Bayer, others from Sofie Biosciences, personal fees from SIRTEX, non-financial support from $\mathrm{ABX}$, personal fees from Adacap, personal fees from Curium, personal fees from Endocyte, grants and personal fees from BTG, personal fees from IPSEN, personal fees from Siemens Healthineers, personal fees from GE Healthcare, personal fees from Amgen, personal fees from Novartis and personal fees from ymabs, outside the submitted work.

\section{Reference}

1. Ambrosini V, Kunikowska J, Baudin E, Bodei L, Bouvier C, Capdevila J, et al. Consensus on molecular imaging and theranostics in neuroendocrine neoplasms. EJC. 2021;146:56-73. https://doi.org/ 10.1016/j.ejca.2021.01.008.

Publisher's note Springer Nature remains neutral with regard to jurisdictional claims in published maps and institutional affiliations. 